Frequency content of current pulses in slapper detonator bridges

K. H. Carpenter

December 21, 2006 
This document was prepared as an account of work sponsored by an agency of the United States Government. Neither the United States Government nor the University of California nor any of their employees, makes any warranty, express or implied, or assumes any legal liability or responsibility for the accuracy, completeness, or usefulness of any information, apparatus, product, or process disclosed, or represents that its use would not infringe privately owned rights. Reference herein to any specific commercial product, process, or service by trade name, trademark, manufacturer, or otherwise, does not necessarily constitute or imply its endorsement, recommendation, or favoring by the United States Government or the University of California. The views and opinions of authors expressed herein do not necessarily state or reflect those of the United States Government or the University of California, and shall not be used for advertising or product endorsement purposes.

This work was performed under the auspices of the U.S. Department of Energy by University of California, Lawrence Livermore National Laboratory under Contract W-7405-Eng-48. 


\title{
Frequency content of current pulses in slapper detonator bridges
}

\author{
Kenneth H. Carpenter \\ June 12, 2006 \\ revised December 20, 2006
}

\begin{abstract}
DFT amplitudes are obtained for digital current pulse files. The frequency content of slapper detonator bridge current pulses is obtained. The frequencies are confined well within the passband of the CVR used to sample them.
\end{abstract}

\section{Contents}

1 Introduction $\quad 1$

2 Obtaining frequency spectra from current pulse data $\quad 2$

2.1 The DFT and its relationship to frequency content of a pulse . . . . . . . . . 2

2.2 Tests for the frequency spectrum of a typical bridge current pulse . . . . . . . 3

2.2.1 The effect of noise and "glitches" on the spectrum . . . . . . . . 5

3 Frequency content in current pulses from the "Norton" series of slapper $\begin{array}{ll}\text { detonator tests } & 6\end{array}$

3.1 Method for obtaining the DFT amplitudes . . . . . . . . . . . 6

\section{Introduction}

When slapper detonator bridges are activated by current pulses from a capacitative discharge fireset, to a first approximation, the current pulse looks like a half sine wave at the ringdown frequency of the fireset-bridge combination. A closer examination shows that the current pulse is far from a half sine wave, but, in most cases, has a duration somewhat shorter than the half sine wave. In any event, the spectral content of the current pulse extends to much higher frequencies than the fundamental frequency of the ringdown.

Knowledge of the frequency content of a bridge current pulse is important for accurate measurement of bridge resistivity. The current viewing resistor (CVR) used to measure the current to the bridge has a calibration that is only valid for frequencies less than some design value. One needs to verify that the observed pulse is within this range or else compensation of the measured current values is required.

The frequency spectrum of a bridge current pulse can also help to determine if skin effect will be a significant factor in current distribution to an array of bridges. 
In the following, the method for obtaining frequency spectra for bridge current pulses will be explained, and results for some typical slapper shots will be given. For all the data examined, the CVR's used were adequate without any compensation.

\section{Obtaining frequency spectra from current pulse data}

Current pulse data is in the form of pairs of time and current values as obtained from a digitizing oscilloscope. The sampling rate varies with different shots. The highest frequency that can be in such data is the Nyquist frequency[1], which is one-half the reciprocal of the sampling interval: $f_{n}=1 /(2 \Delta t)$. Any higher frequencies in the signal should have been removed by an analog anti-aliasing filter before the data was sampled. The frequency spectrum of a signal is then obtained by applying a discrete Fourier transform (DFT) to the sampled data.

\subsection{The DFT and its relationship to frequency content of a pulse}

Given a finite sequence of values, $x_{n}, n=1 \ldots N$, the DFT of this sequence is given by the sequence, $y_{m}, m=1 \ldots N$, where

$$
y_{m}=\sum_{n=1}^{N} x_{n} e^{-j 2 \pi(n-1)(m-1) / N} .
$$

In eq.(1), $j=\sqrt{-1}$. The inverse DFT is given by

$$
x_{n}=\frac{1}{N} \sum_{m=1}^{N} y_{m} e^{+j 2 \pi(n-1)(m-1) / N} .
$$

When one has a periodic function, $g(t)=g(t+T)$, it may be represented by a Fourier series, in complex form,

$$
g(t)=\sum_{k=-\infty}^{+\infty} G_{k} e^{j 2 \pi k f t},
$$

where $f=1 / T$ is the fundamental frequency. The Fourier coefficients are given by

$$
G_{k}=f \int_{0}^{\frac{1}{f}} g(t) e^{-j 2 \pi k f t} d t .
$$

When the sequence $x_{n}$ is obtained by sampling $g(t)$ the relationship between the $y_{m}$ and $G_{k}$ values is approximately given by replacing the integral in eq.(4) with a sum:

$$
G_{k}=f \int_{0}^{\frac{1}{f}} g(t) e^{-j 2 \pi k f t} d t \approx \frac{1}{T} \sum_{n=0}^{\frac{T}{\Delta t}-1} g(n \Delta t) e^{-j 2 \pi k f n \Delta t} \Delta t=\frac{1}{N} y_{k+1},
$$

where $N=T / \Delta t$. Thus the DFT values $y_{m}$ correspond to the number of samples $N$ times the complex Fourier series coefficients for the same harmonic.

Note that the definition of $y_{m}$ in eq.(2) has $y_{m}$ periodic in $m$ with period $N$. Thus the negative values for $k$ in $G_{k}$ may be related to positive $m$ values for $y_{m}$. Note also that for $g(t)$ a real signal, the $G_{k}$ and $y_{m}$ values are complex, and $G_{k}=G_{-k}^{*}$ - negating the subscript is equivalent to taking the complex conjugate. Thus $G_{-k}=y_{k+1}^{*}=y_{N-k+1}$. 
To put the $y_{m}$ coefficients in terms of the more familiar harmonic amplitudes, note that for the $k^{\prime}$ th harmonic in the Fourier series the total contribution from eq.(3) is

$$
\begin{aligned}
G_{-k} e^{-j 2 \pi k f t}+G_{k} e^{+j 2 \pi k f t}=2 \operatorname{Re}\left[G_{k} e^{j 2 \pi k f t}\right] & =2\left[\operatorname{Re}\left(G_{k}\right) \cos (2 \pi k f t)-\operatorname{Im}\left(G_{k}\right) \sin (2 \pi k f t)\right] \\
& =\frac{2}{N}\left|y_{k+1}\right| \cos \left(2 \pi k f t-\phi_{k+1}\right)
\end{aligned}
$$

where $\phi_{k+1}$ is the phase angle of $y_{k+1}$ in polar form. Thus the amplitude of the $n$th harmonic of fundamental frequency $f$ in the signal $g(t)$ is given by $\frac{2}{N}\left|y_{n+1}\right|$. The DC component in $g(t)$ is $\frac{1}{N} y_{1}$.

\subsection{Tests for the frequency spectrum of a typical bridge current pulse}

In order to examine the effects of the approximation inherent in the conversion of the integral in eq.(4) to a sum of $N$ terms in a sampled data representation and of the assumption that the sampled signal is periodic, several DFT evaluations were made on the current data for a single bridge current pulse. (The current pulse used was for "Norton" shot number 1071.) The sampled data of the current pulse is shown in Fig. 1. A plot of the current pulse versus time for this shot is shown in Fig. 2. The plot shows the current as a continuous curve, but there were 900 samples in the data plotted with $\Delta t=2 \mathrm{~ns}$.

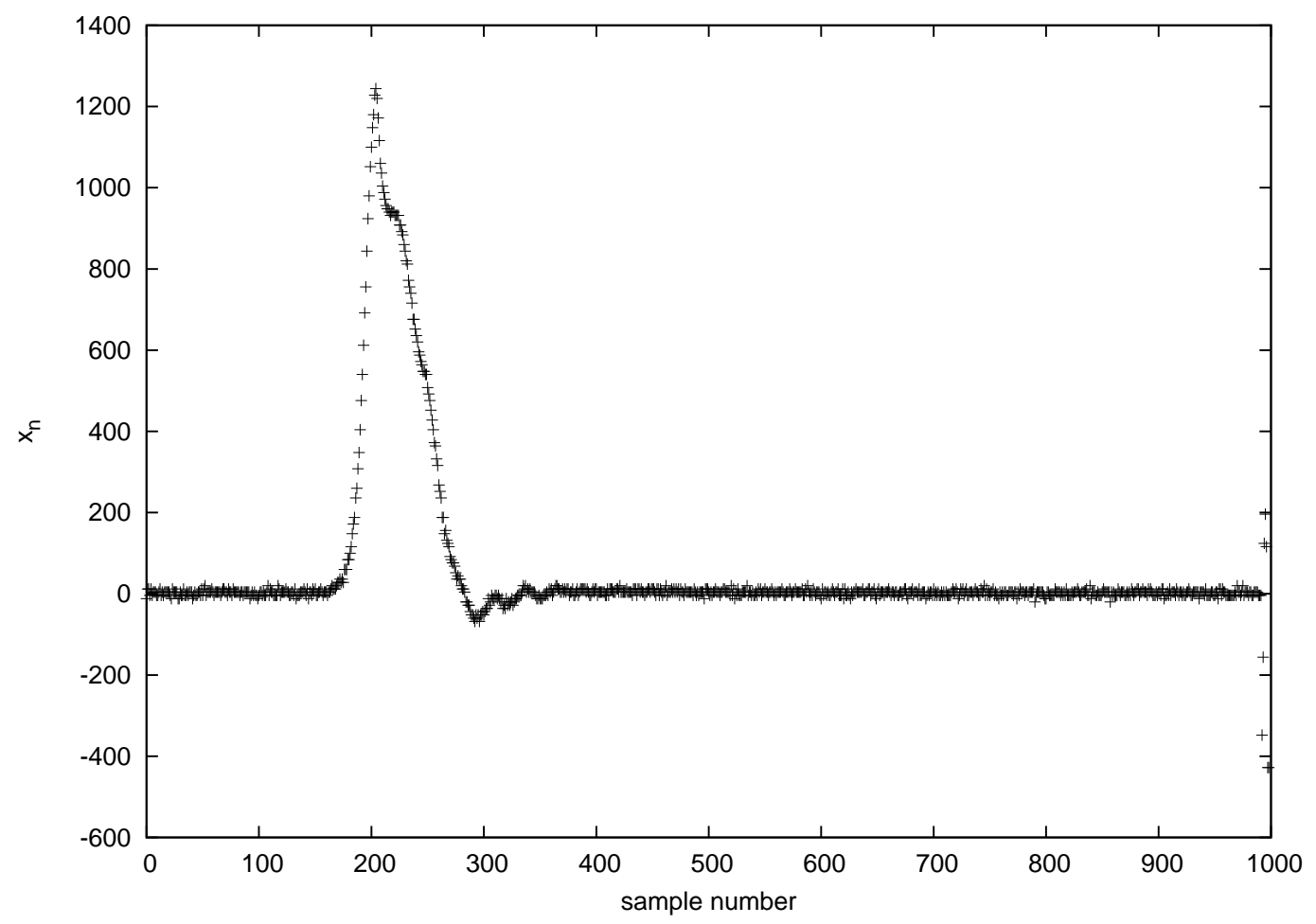

Fig. 1: Current samples for test pulse. Sample interval was $2 \mathrm{~ns}$

From Fig. 1 one sees that there is a "glitch" near the end of the displayed time in the current trace. It is a cycle or so of oscillation at the sampling frequency. This is clearly not part of the actual current pulse, so this end of the sampled data was truncated, and only the first 900 samples used in obtaining DFT's. 


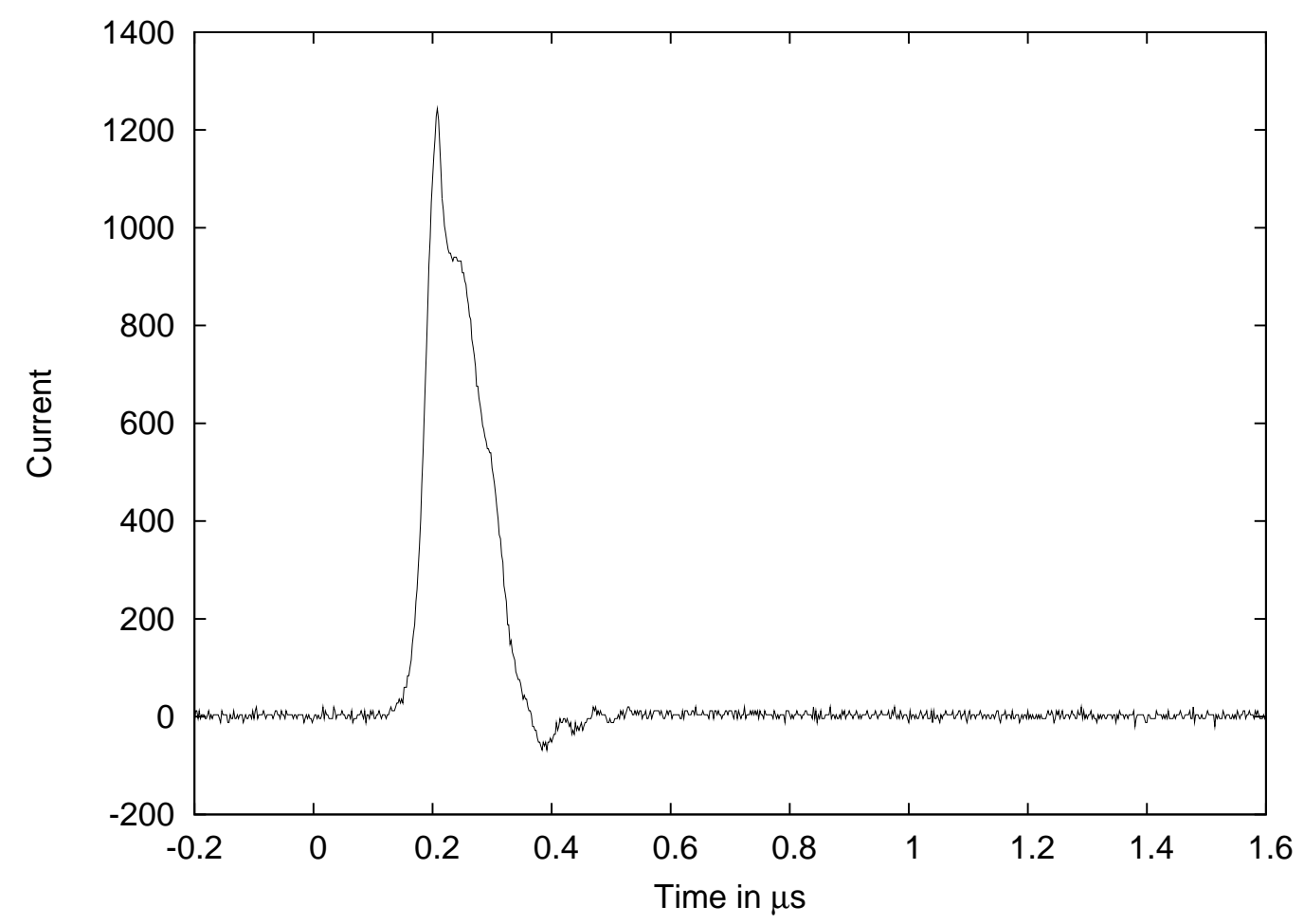

Fig. 2: Current versus time for test pulse. Trailing 100 samples have been removed before plotting.

Since the current pulse is not actually a periodic function, but is only a single shot event, to approach the actual case in a DFT one must add many addition samples of zero either before or after the given 900 samples to approach the ideal case. This increases the $N$ value in the sums for the DFT and increases the number of coefficients correspondingly. The fundamental frequency $f=1 /(N \Delta t)$ decreases for larger $N$. Thus the result of larger $N$ is to have a finer resolution of the frequency content of the pulse. When the frequency resolution is small, so that individual $y_{m}$ values would not be separated on a plot, the area under the curve for $y_{m}$ versus $f$ should be the same for equal increments $\Delta f$, centered on the same $f$ value, if two different $N$ values give the same spectrum. This is because the coefficients of a single harmonic go as $1 / N$ but the number of such coefficients in a range $\Delta f$ goes as $N$. Another way to explain this is to note that adding zero padding does not change the $y_{m}$ value corresponding to a particular frequency. Fig. 3 shows plots for $\left|y_{m}\right|$ versus $f$ for DFT's of the test pulse with the first 900 current data points padded with zeros at the end to give total $N$ values of 1000, 2000, and 4000 . (The plots in Fig. 3 show a frequency range from zero to the Nyquist frequency. No higher frequency amplitude information is available from this sampled data.) From Fig. 3 one can see that there is no observable change in the spectrum on adding additional zero padding beyond the 1000 point case. However, if one takes a magnified look at a portion of the spectrum, as shown in Fig. 4, one can see how increasing the number of samples by zero padding adds detail to the spectrum. 


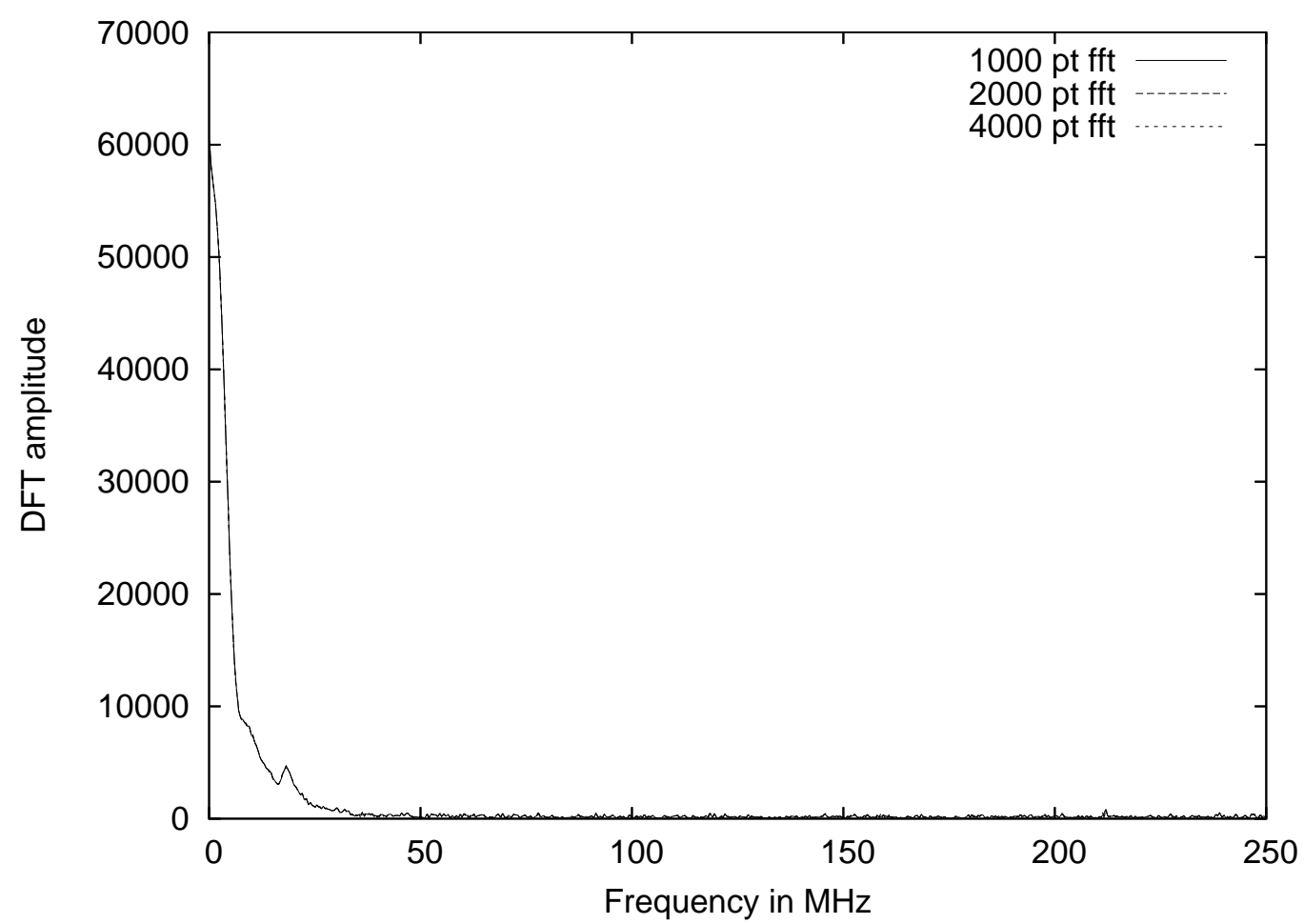

Fig. 3: Magnitude of Fourier components for the current pulse in Fig. 1 with zero padding to different total lengths for DFT evaluation.

\subsubsection{The effect of noise and "glitches" on the spectrum}

There is no significant content in the spectrum in Fig. 3 for frequencies above $50 \mathrm{MHz}$. However there is noise in the spectrum from 50 to $250 \mathrm{Mhz}$. This noise in the DFT can be eliminated by smoothing the sampled data (using DerSmooth) before taking the DFT. This is shown by the smoothed data in Fig. 5 and its DFT amplitude in Fig. 6.

An alternate approach to reducing the noise in the DFT is to truncate the sections of the data before and after the signal - in this case restricting the data used to samples 150 to 400 . This does not remove as much of the noise in the spectrum as smoothing the data does, as there is still noise on the samples from 150 to 400. This may also be seen in Fig. 6. The frequency range in Fig. 6 has been restricted to show the details in the effect of smoothing on the spectrum. A plot of the full range shows the spectrum is essentially the same outside of the noise region, but some details are changed by the smoothing or truncation of noise segments.

When the "glitch" at the end of the sampled data is not truncated the spectrum shows many more high frequency components. This is shown in Fig. 7. When compared to Fig. 3 for the truncated data, one sees that the spectrum of the data with the "glitch" at the end has significantly more content in the range of 50 to $250 \mathrm{MHz}$. Thus it is important to remove such spurious data before taking the DFT. 


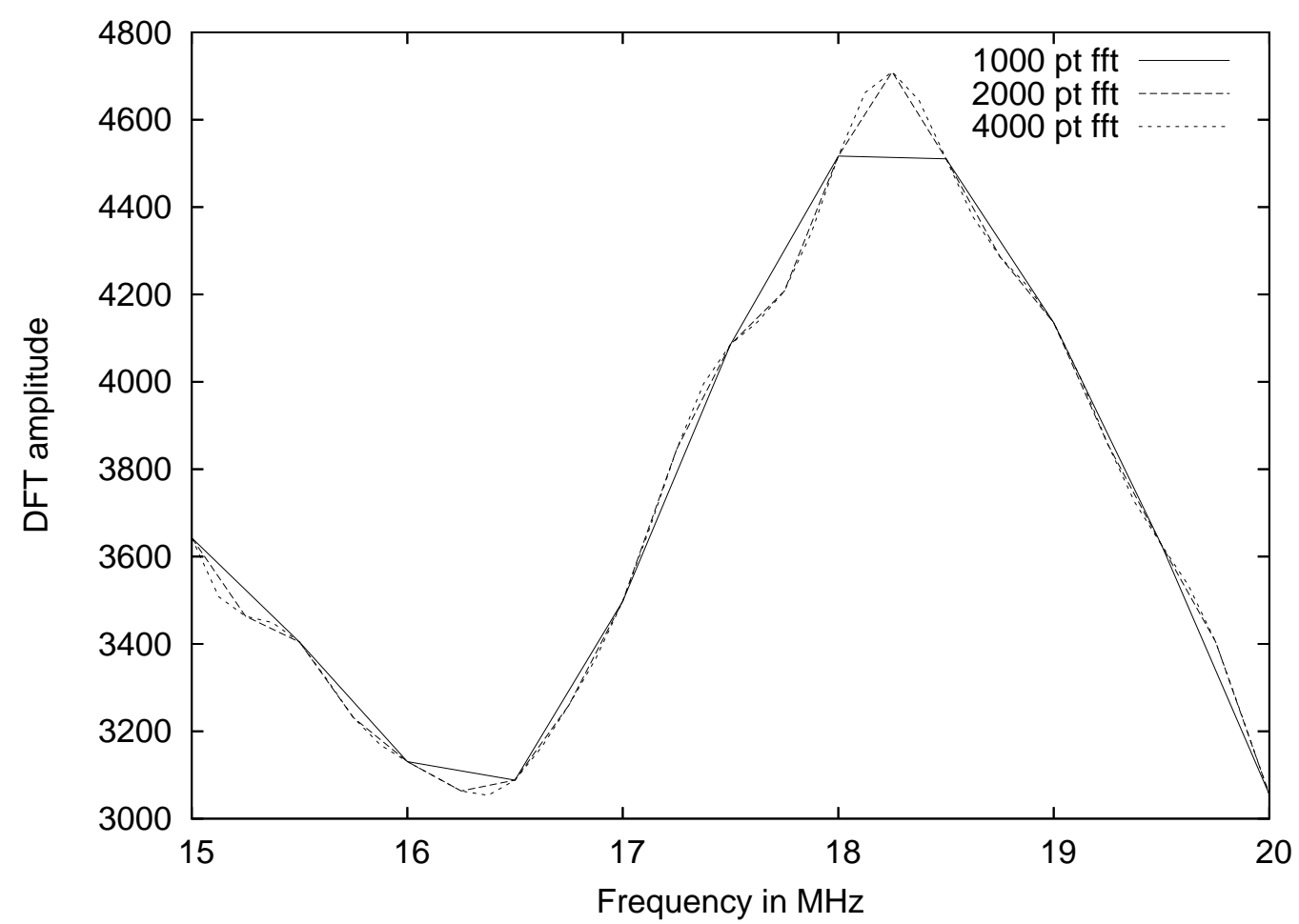

Fig. 4: Magnitude of Fourier components for the current pulse in Fig. 1 with zero padding to different total lengths for DFT evaluation. Restricted frequency range displayed to show the effect of different amounts of padding.

\section{Frequency content in current pulses from the "Norton" series of slapper detonator tests}

A DFT has been obtained for a representative set of the current pulses in the Norton series of slapper detonator bridge firings. All of the data was acquired using a T\&M Research Products, Model W-2-005C-1FC, $0.005 \Omega$ current viewing resistor (CVR)[2]. The manufacturer's specifications for this CVR[3] give its bandwidth as $200 \mathrm{MHz}$. The DFT amplitudes obtained show that the CVR was used well within its specifications.

\subsection{Method for obtaining the DFT amplitudes}

Since a large number of similar current data files required identical processing to obtain their frequency content, a script was written to use Octave[4] (or MATLAB[5]) to carry out this task in an efficient manner. The DFT was obtained using the FFT algorithm built into the Octave code. In order to make the DFT amplitudes directly comparable between shots with different current magnitudes, the amplitudes are first normalized by the DC value before saving.

The Norton data available for analysis has shot numbers from 1071 through 1108. Shots 1071 through 1088 have 1000 samples while shots 1089 through 1108 have 2500 samples. Shots 1071 through 1088 and 1090 through 1094 use a sampling rate of $\Delta t=2$ ns; shot 1089 has $\Delta t=4 \mathrm{~ns} ;$ shots 1095 through 1100 have $\Delta t=0.4 \mathrm{~ns}$; shots 1101 through 1108 have $\Delta t=0.2 \mathrm{~ns}$. From these, the DFT values were obtained for shot numbers 1071, 1074, 1088, 1089, 1090, 1092, 


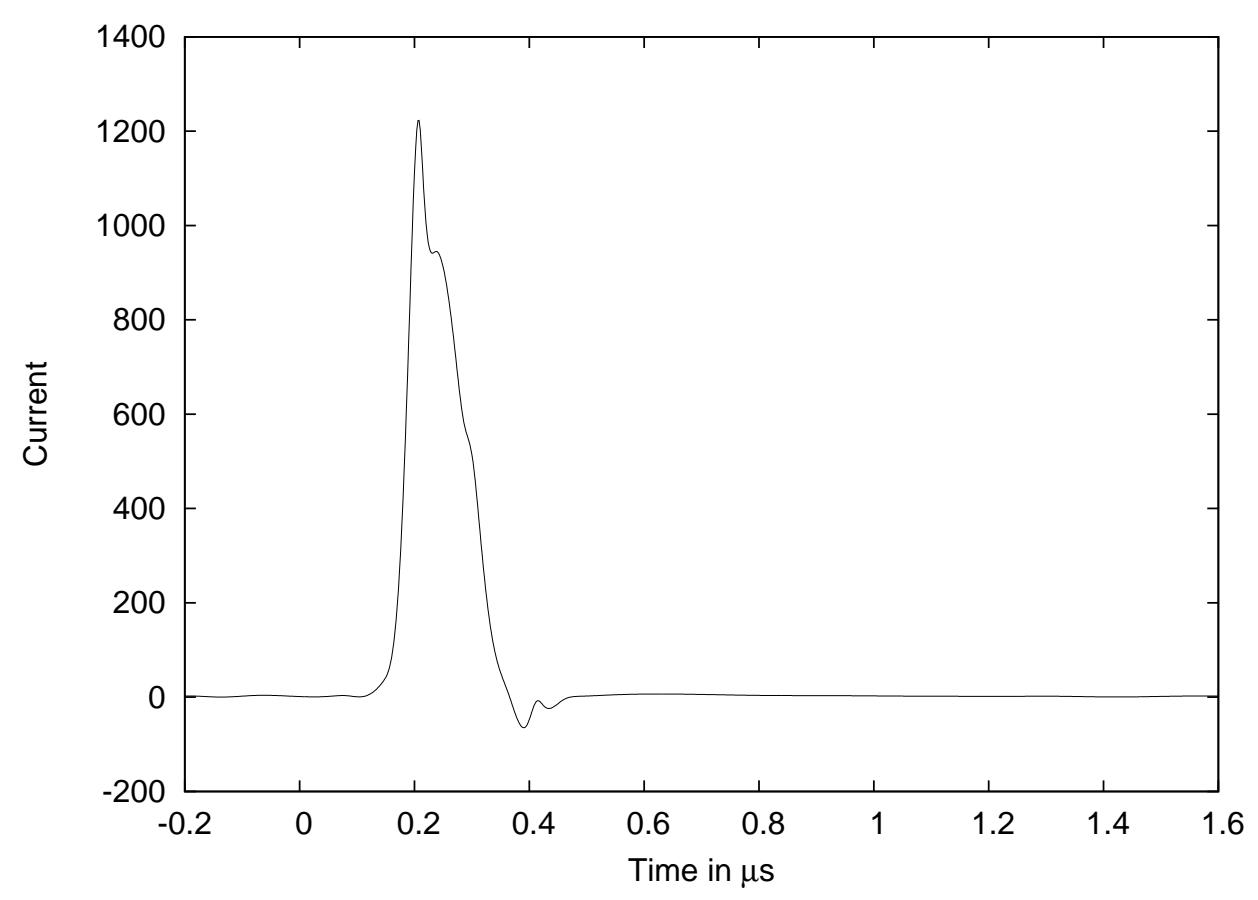

Fig. 5: Current versus time for test pulse of Fig. 2 after smoothing with DerSmooth code with smooth value -1 . Trailing 100 samples have been removed before plotting.

$1094,1095,1098,1100,1101,1105$, and 1108. Some of the shots acquired the current data on two different digitizer channels. The data from the two channels is the same for the current pulses, but will have different noise components. The DFT amplitudes from the two channels of the same current are also the same except for the noise component. Thus only one of the two has been used in the following. (The exception is shot 1101, where the two channels have different currents - one clips on the positive side the other clips on the negative side. Both DFT's are plotted for these channels.)

The DFT amplitudes are plotted versus frequency in two groups, the first having $\Delta t$ of 2 and $4 \mathrm{~ns}$ and the second having $\Delta t$ of 0.2 and $0.4 \mathrm{~ns}$. All DFT data plotted is limited to the range of zero to the Nyquist frequency. The lower sampling rate DFT data in Fig. 8 shows no frequency components of significance above $50 \mathrm{MHz}$. The higher sampling rate DFT data in Fig. 9 while allowing for a higher Nyquist frequency, still shows no significant frequency components above $50 \mathrm{MHz}$. In fact, even the noise components above $500 \mathrm{MHz}$ are so small that the plot was truncated for higher frequencies in order to show more detail at the lower frequencies.

\section{References}

[1] Rodger E. Ziemer, William H. Tranter, D. Ronald Fannin, Signals and Systems: Continuous and Discrete, Macmillan Publishing Co., Inc., New York, N.Y., 1983. 


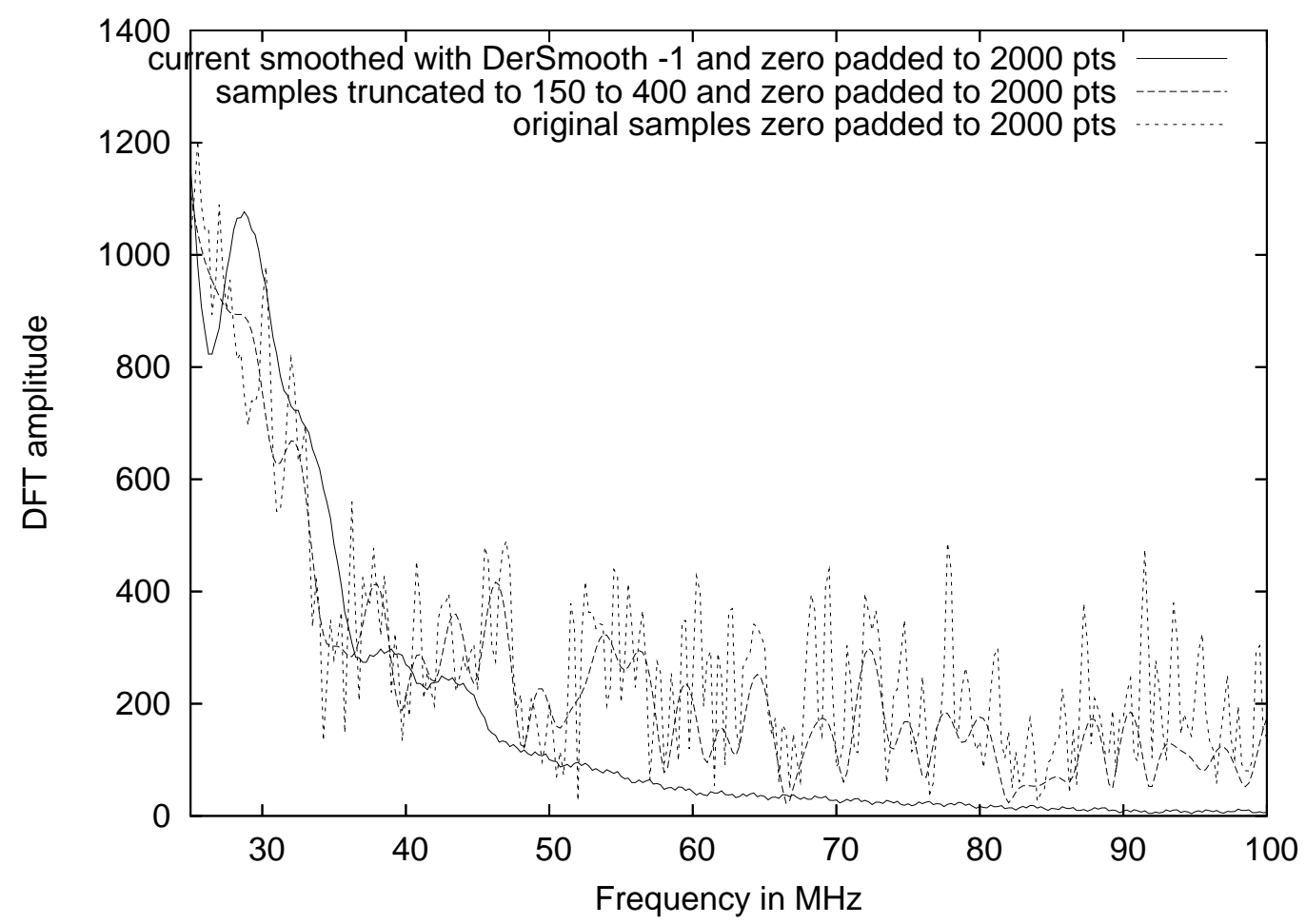

Fig. 6: Magnitude of Fourier components for the smoothed current pulse in Fig. 5 with zero padding to 2000 samples, compared to the Fourier components for the unsmoothed current pulse with and without removal of leading and trailing noise sections. Restricted frequency range displayed to show the effects more clearly.

[2] Roy L. Hanks, Lawrence Livermore National Laboratory, private communication.

[3] T \& M Research Products, Albuquerque, NM. http://www.tandmresearch.com/

[4] John W. Eaton, GNU Octave: A high level language for numerical computations, 3rd Ed., February 1997. Available from http://www.gnu.org/software/octave/docs.html.

[5] MATLAB囚High-Performance Numeric Computation and Visualization Software, User's Guide, The Math Works, Inc., 1992. Newer documentation is available at http://www. mathworks.com/ 


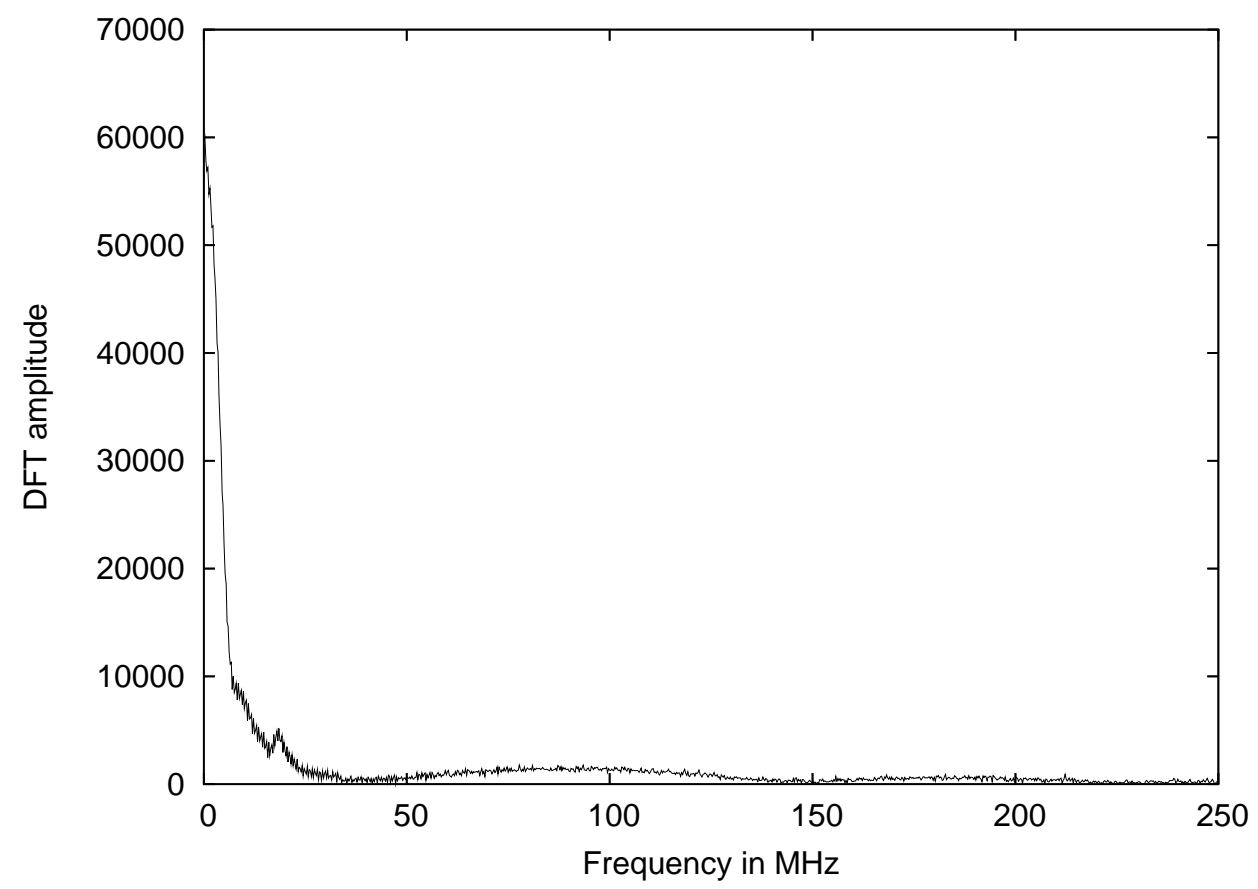

Fig. 7: Magnitude of Fourier components for the all the samples in Fig. 1, padded to zeros to 2000 samples. 


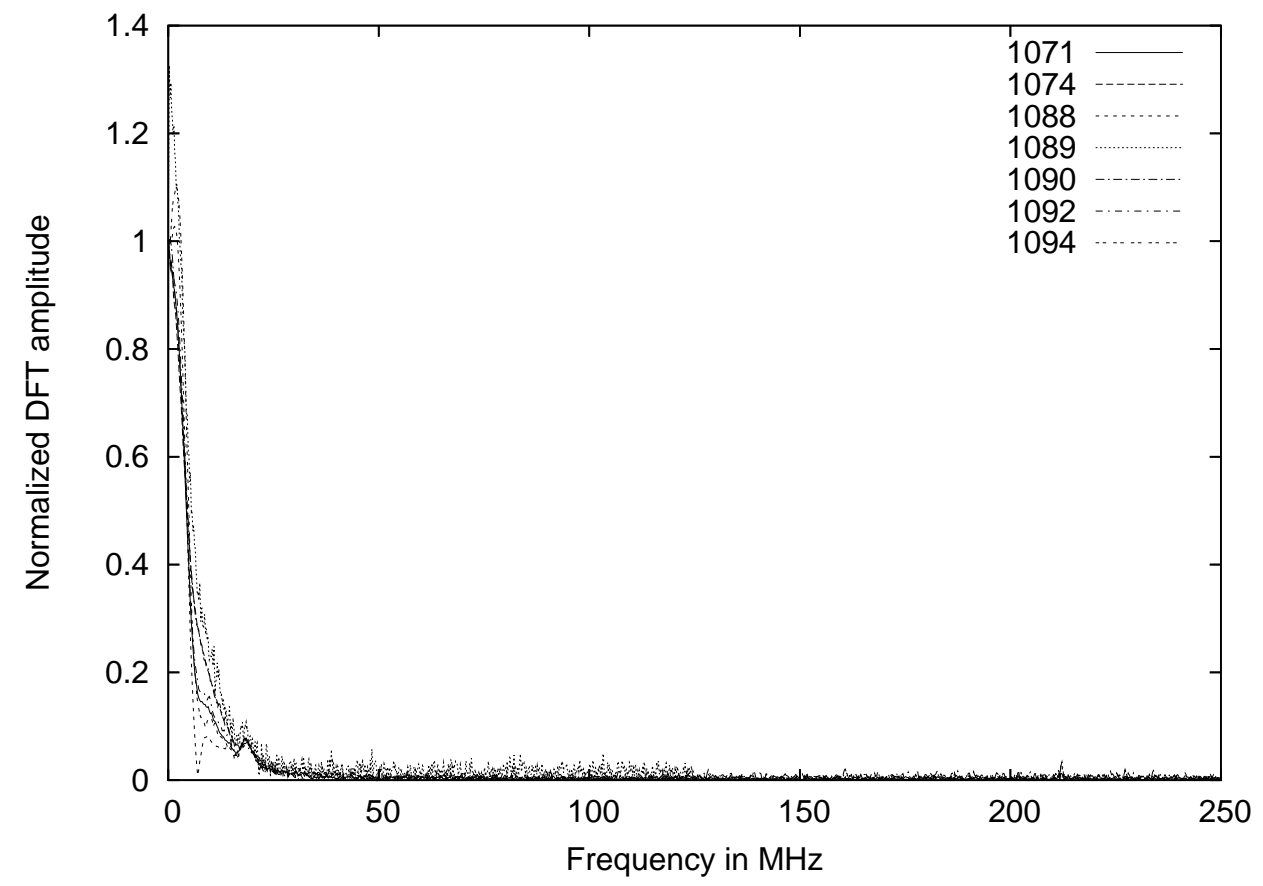

Fig. 8: Magnitude of Fourier components for selected current pulse data from the "Norton" series having time sampling at $2 \mathrm{~ns}$ or $4 \mathrm{~ns}$. 


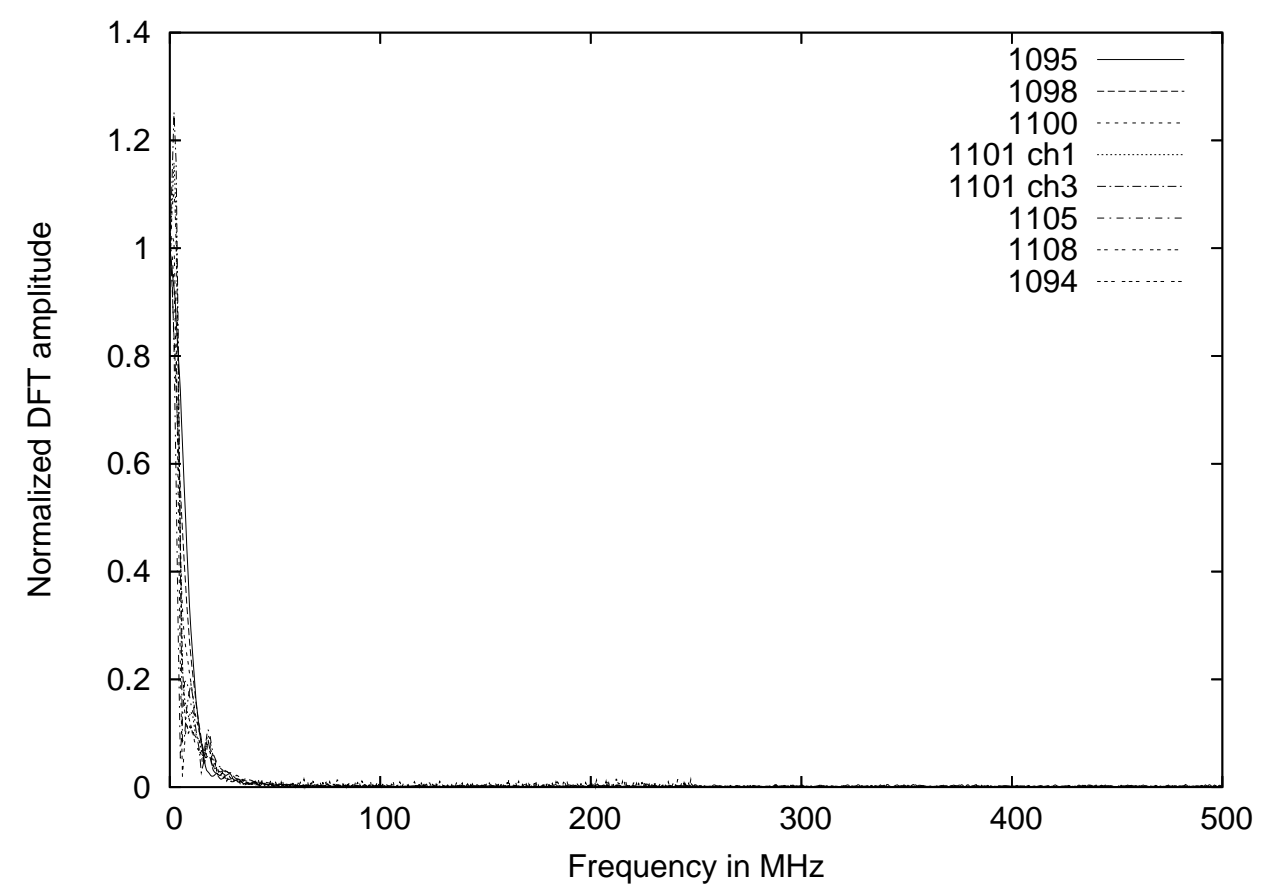

Fig. 9: Magnitude of Fourier components for selected current pulse data from the "Norton" series having time sampling at $0.2 \mathrm{~ns}$ or $0.4 \mathrm{~ns}$. 